\title{
Cannabis alleviates neuropathic pain and reverses weight loss in diabetic neuropathic cachexia in a previous heroin abuser
}

\author{
Deeb Daoud Naccache \\ Institute of Endocrinology and the Centre for Excellence in Diabetes and Obesity, Rambam Health Care campus, \\ Haifa, Israel \\ Correspondence \\ should be addressed \\ to D Daoud Naccache \\ Email \\ d_deeb@rambam.health. \\ gov.il
}

\section{Summary}

Ten years after the successful withdrawal from heroin abuse, a person with diabetes suffered intractable pain and severe muscular emaciation consistent with the syndrome of diabetic neuropathic cachexia. Anti-neuropathic medications failed neither to alleviate suffering and reverse weight loss, nor to stop muscular emaciation. Vigilant evaluation for weight loss aetiologies revealed no responsible aetiology. Prescribing medical cannabis became mandatory, with the intention to alleviate neuropathic pain, regain muscular mass and strengthen legs, enable standing upright and walking normally. Medical cannabis for pain-relief, and the orexigenic properties of tetrahydrocannabinol (THC) ingredient successfully achieved these goals.

\section{Learning points:}

- Medical cannabis can serve to promptly alleviate severe diabetic neuropathic pain.

- Past history of heroin abuse was not an absolute contraindication to medical cannabis use.

- Medical cannabis increased appetite and reversed muscular emaciation.

- Medical cannabis decreased chronic pain and hence, its catabolic consequences.

\section{Background}

Weight loss, muscular emaciation, loss of appetite and a depressive mood are a few of the leading features of the daily functional status of rare cases of diabetic neuropathic cachexia (DNC).

Our patient, who succeeded in heroin withdrawal a decade earlier, posed a unique challenge - using medical cannabis was the last resort for treating severe DNC, as it had an undetermined risk for potentially new substance abuse. A similar case, that we previously reported, presented a case of overlooked acromegaly, perplexed with toxic iodide-overload thyroid adenoma and uncontrolled diabetes. This complexity posed challenges to therapeutic goal priorities (1).

Medicinal cannabis clinical experiences and legal use have emerged during the last few decades (2). For its pain-relieving and appetite-stimulating properties (3), we found cannabis suitable for tackling severe neuropathic pain, severe muscular emaciation, anorexia, disturbed sleep patterns and changes in the patient's mood.

\section{Case presentation}

An Arab male aged 62, was referred to our clinic by his community-based neurologist, to apply for a licence for the 'handling and use' of medical cannabis in accordance with the regulations of the Israeli Medical Cannabis Unit in the Ministry of Health. The use of Pregabalin and Duloxetine had proved inefficient. An opioid oral painkiller (Tramadol) caused intolerable dizziness and instability. 
The patient walked slowly into the clinic room with a waddling gait and was given a helping hand from his wife. His face expressed his agony and a desperate need for help.

A short physical examination revealed severely atrophied anterior and posterior thigh muscles. A suspicion of DNC was quickly identified, and the patient was referred for a complete in-hospital weight loss investigation.

The history of the patient revealed:

Heroin abuse until 10 years prior to his admission.

Attempted suicide.

Controlled essential hypertension.

Benign hypertrophy of prostate without obstruction.

Tobacco use since the age 15 years.

Mild chronic restrictive pulmonary disease.

Diabetes mellitus since July 2007, uncontrolled in the past.

An appendectomy.

See Table 1 for recent medications.

\section{Investigation}

Upon admission to an internal department, the patient complained of a symmetrical gloves-and-socks pattern of neuropathic pain; pronounced bilateral leg weakness; pedal anaesthesia; disturbed sleep disorder and depressed mood. His pain was registered as $7-8 / 10$ on a visual analogue scale (VAS).

During the last year, the patient suffered diffuse central abdominal pain, unassociated with meals or toilet habits. He lost his appetite and $25 \mathrm{~kg}$ of body weight (30\% of $84 \mathrm{~kg}$ at baseline). In addition, the patient complained of recent orthostatic dizziness and he fainted once after standing up quickly.

Table 1 Recent medications.

\begin{tabular}{l}
\hline Medication \\
\hline Duloxetine \\
Duloxetine \\
Pregabalin \\
Metformin \\
Enalapril \\
Atorvastatin \\
Acetylsalicylic acid \\
Omeprazole \\
Salbutamol Sulphate \\
Dutasteride $500 \mu g$ and \\
Tamsulosin $\mathrm{HCl} 400 \mu \mathrm{g}$ \\
\hline
\end{tabular}

\begin{tabular}{lll}
\hline Dosage & & Per day \\
\cline { 1 - 1 } $60 \mathrm{mg}$ & & Twice \\
$30 \mathrm{mg}$ & & Once \\
$75 \mathrm{mg}$ & & Twice \\
$850 \mathrm{mg}$ & Twice \\
$5 \mathrm{mg}$ & Once \\
$40 \mathrm{mg}$ & Once \\
$100 \mathrm{mg}$ & Once \\
$20 \mathrm{mg}$ & Once \\
$0.1 \mathrm{mg}$ & Inhalation as needed \\
$1 \mathrm{tablet}$ & Once \\
& \\
\hline
\end{tabular}

Laboratory studies: serum carcinoembryonic antigen, carcinogenic antigen-125 and 19-9, alpha-fetoprotein, beta-human chorionic gonadotropin, prostatic specific antigen were all within normal limits.

Physical examination: cachectic features, stable hemodynamic and respiratory status. Blood pressure: 113/73 mmHg; Pulse: regular 96 b.p.m.; Temperature: $36.6^{\circ} \mathrm{C}$; Weight: $61 \mathrm{~kg}$; Height: $181 \mathrm{~cm}$; Body surface area $1.79 \mathrm{~m}^{2}$; BMI: $18.6 \mathrm{~kg} / \mathrm{m}^{2}$; Oxygen saturation: $96 \%$; Temporal muscle wasting noted; No lymphadenopathy observed; Heart, lungs: no pathologic findings; Mild diffuse abdominal tenderness, without signs of acute abdomen.

Thigh circumference 10 and $15 \mathrm{~cm}$ above the patella: 35 and $38 \mathrm{~cm}$, respectively. Gastroscopy during hospitalisation revealed antral gastritis. The patient was instructed to comply strictly with the regular use of a proton pump inhibitor (Omeprazole $20 \mathrm{mg}$ qd), instead of his habit of using it intermittently. A weight-loss investigation that commenced 6 months earlier, revealed a normal gastroscopy and colonoscopy. CT of neck, chest, abdomen, and pelvis revealed clinically insignificant findings in the chest and abdomen.

Laboratory tests are summarised in Table 2.

\section{Treatment}

The application for a medical cannabis licence, supported with a psychiatric opinion stating that there was no future potential for the risk of cannabis abuse, was approved, and the patient started on oil extract blend of Sativa and Indica cannabis, containing a THC to Cannabidiol ratio of T10:C10, of $20 \mathrm{~mL}$ per month.

\section{Outcome and follow-up}

Four months after starting with medical cannabis, the patient reported improved appetite while regaining $10 \mathrm{~kg}$ of body weight. He was enjoying a peaceful night's sleep, improved strength in his legs and decreased pain, VAS was down to $2 / 10$, while pain level without cannabis was VAS $6-7 / 10$. His daily cannabis dosage varied between three and five drops of oil extract, according to his suffering. When asked about the risk of abuse, the patient expressed no craving to increase the dosage, and he did not feel stressed when he was away from home. He could tolerate some increase in pain until arriving back home and then he only had to take one drop more than the usual dose. His score was a 'zero' for the four questions in the CAGE four questions form Adapted to Include Drugs (CAGE-AID) questionnaire (4), meaning: he is not at risk of cannabis 
Table 2 Laboratory tests: recent findings.

\begin{tabular}{|c|c|c|}
\hline Parameters & Result & Normal range \\
\hline HbA1c, \% & 6.6 & $4-5.7$ \\
\hline Sodium, mmol/L & 140 & $133-145$ \\
\hline Potassium, mmol/L & 4.4 & $3.5-5.3$ \\
\hline Creatinine, mg/dL & 0.86 & $0.3-0.7$ \\
\hline C-reactive protein, mg/dL & 0.2 & $0-0.5$ \\
\hline Uric acid, mg/dL & 2.26 & $3.5-7.5$ \\
\hline Albumin, g/dL & 4.3 & $3.2-4.6$ \\
\hline LDL cholesterol, mg/dL & 137 & $75-140$ \\
\hline Haemoglobin, g/dL & 15.4 & $13-17$ \\
\hline White blood cells, k/ $\mu \mathrm{L}$ & 8.69 & 4-10.8 \\
\hline TSH, mIU/L & 0.723 & $0.27-4.2$ \\
\hline HIV test & Negative & \\
\hline
\end{tabular}

$\mathrm{TSH}$, thyroid stimulating hormone.

dependence. The patient found that a $20 \mathrm{~mL}$ vial of the blend T10:C10 oil extract, was sufficient for a month.

\section{Discussion}

DNC is a rare complication of painful diabetic sensory neuropathy. In a previously reported case of DNC, we raised the notion that pain in itself, induces a catabolic state which might lead to sarcopenia, via an inflammatory process similar to that evoked in the wound healing process (5). Pain dysregulates diabetic control (6), via the induction of insulin resistance and increasing endogenous glucose output (hepatic in source).

Our case presented a unique challenge and concern. The patient was a previous abuser of heroin; therefore, the administration of medical cannabis was a formidable one, due to concerns as to the potential abuse of cannabis by the patient. However, cannabis was our last resort to control the neuropathic pain.

We decided to prescribe cannabis, dependant on (i) a deliberate opinion of his named psychiatrist that there was a negligible risk of abuse; (ii) the appetite-mediated beneficial anabolic effect of the THC component; (iii) pain control.

The anabolic effect was plausible by increasing the insulin dose; however, insulin proved inefficacious in pain control. It is a fact that the state of good diabetic control hampers increasing insulin dose for anabolic purposes, due to the risk of insulin-induced hypoglycaemia. Cannabis might interact with glucose homeostasis, beyond that of inducing insulin resistance and increasing endogenous glucose production, mentioned earlier in this discussion. It was of some interest that the appetitive properties of cannabis were observed with changes in blood levels of ghrelin - the hormone of hunger, and not that of insulin (7).

In fact, the improved appetite, weight gain, sleep quality, as well as the better moods that our patient experienced while taking medical cannabis, proved that the clinical rationale and decision in administrating cannabis were adequate and successful.

We find this clinical experience worthy of publication and beneficial for both patients and their caregivers to consider cannabis in similar situations.

As well as severe sensory-motor diabetic neuropathy, our case shares autonomic dysfunction complaints with other reported cases. The organic impotence and a fainting bout after standing up quickly is in line with an orthostatic disorder.

To the best of our knowledge, this is the first case to report the promising use of medical cannabis in a catabolic state, notwithstanding its controversial effect on conditions of sarcopenia and muscle emaciation in cancer and HIV patients (8).

If pain management comes to a dead end, either because of inefficient methods or having unbearable side effects, then, in the presence of weight loss and muscular emaciation, medical cannabis is a predictably good therapeutic alternative.

\section{Patient's perspective}

It was my elderly mother who crawled into my house, 'begging' me to get this last chance and start cannabis. I found more than mere cannabis recommendation, as several unanswered questions till today, got amazing answers. It was as if I found a treasure. My self-confidence returned after several months of living like a zombie.

\section{Declaration of interest}

The author declares that there is no conflict of interest that could be perceived as prejudicing the impartiality of the research reported.

\section{Funding}

This research did not receive any specific grant from funding agencies in the public, commercial or non-profit sectors.

\section{Patient consent}

We confirm that a written consent form has been obtained from the patient for the publication of the submitted article and accompanying images.

\section{Author contribution statement}

Deeb Daoud Naccache, who made the tentative diagnosis and treatment, drafted this case report and continues to be the patient's endocrinologist. 


\section{References}

1 Naccache DD, Nseir WB, Herskovitz MZ \& Khamaisi MH. Diabetic neuropathic cachexia: a case report. Journal of Medical Case Reports 20148 20. (https://doi.org/10.1186/1752-1947-8-20)

2 Romero-Sandoval EA, Fincham JE, Kolano AL, Sharpe BN \& Alvarado-Vázquez PA. Cannabis for chronic pain: challenges and considerations. Pharmacotherapy 201838 651-662. (https://doi. org/10.1002/phar.2115)

3 Riggs PK, Vaida F, Rossi SS, Sorkin LS, Gouaux B, Grant I \& Ellis RJ. A pilot study of the effects of cannabis on appetite hormones in HIVinfected adult men. Brain Research 20121431 46-52. (https://doi. org/10.1016/j.brainres.2011.11.001)

4 Brown RL \& Rounds L. Conjoint screening questionnaires for alcohol and drug abuse: criterion validity in a primary care practice. Wisconsin Medical Journal 199594 135-140.

5 Desborough JP. The stress response to trauma and surgery. British Journal of Anaesthesia 200085 109-117. (https://doi.org/10.1093/ bja/85.1.109)

6 CDC. 10 Surprising things that can spike your blood sugar, 2020. (available at: https://www.cdc.gov/diabetes/library/spotlights/blood-s ugar.html)

7 Farokhnia M, McDiarmid GR, Newmeyer MN, Munjal V, Abulseoud OA, Huestis MA \& Leggio L. Effects of oral, smoked, and vaporized cannabis on endocrine pathways related to appetite and metabolism: a randomized, double-blind, placebo-controlled, human laboratory study. Translational Psychiatry 202010 71. (https://doi. org/10.1038/s41398-020-0756-3)

8 Mücke M, Weier M, Carter C, Copeland J, Degenhardt L, Cuhls H, Radbruch L, Häuser W \& Conrad R. Systematic review and metaanalysis of cannabinoids in palliative medicine. Journal of Cachexia, Sarcopenia and Muscle 20189 220-234. (https://doi.org/10.1002/ jcsm.12273)

Received in final form 16 August 2020

Accepted 18 September 2020 